\title{
Unplanned reinterventions are associated with postoperative mortality in neonates with critical congenital heart disease
}

\author{
Mjaye L. Mazwi, MD, ${ }^{a}$ David W. Brown, MD, ${ }^{\text {a }}$ Audrey C. Marshall, MD, ${ }^{\text {a }}$ Frank A. Pigula, MD, \\ Peter C. Laussen, MBBS, ${ }^{\mathrm{a}}$ Angelo Polito, MD, MPH, ${ }^{\mathrm{a}}$ David Wypij, $\mathrm{PhD},{ }^{\mathrm{a}, \mathrm{c}, \mathrm{d}}$ and \\ John M. Costello, MD, MPH
}

\begin{abstract}
Objective: Neonates with critical congenital heart disease remain at risk of adverse outcomes after cardiac surgery. Residual or undiagnosed anatomic lesions might be contributory. The present study aimed to describe the incidence and type of cardiac lesions that lead to early, unplanned cardiac reintervention, identify the risk factors for unplanned reintervention, and explore the associations between unplanned reinterventions and hospital mortality.
\end{abstract}

Methods: The present single-center retrospective cohort study included 943 consecutive neonates with critical congenital heart disease who underwent cardiac surgery from 2002 to 2008. An unplanned cardiac reintervention was defined as a cardiac reoperation or interventional cardiac catheterization performed during the same hospitalization as the initial operation. Multivariate logistic regression analyses were used to identify the risk factors for unplanned cardiac reintervention and hospital mortality.

Results: Of the 943 neonates, 104 (11\%) underwent an unplanned cardiac reintervention. The independent predictors of unplanned reintervention included prenatal diagnosis, lower birth weight, need for mechanical ventilation before the initial cardiac operation, lower attending surgeon experience, and greater Risk Adjustment in Congenital Heart Surgery, version 1, category. Those who underwent reintervention had increased hospital mortality $(\mathrm{n}=33 / 104,32 \%)$ relative to those who did not $(\mathrm{n}=31 / 839,4 \%$; adjusted odds ratio, $8.6 ; 95 \%$ confidence interval, 4.7 to $15.6 ; P<.001)$. The mortality rates among patients undergoing surgical reintervention $(23 / 66,35 \%)$ or transcatheter reintervention $(4 / 16,25 \%)$, or both $(6 / 22,27 \%)$ were similar $(P=.66)$.

Conclusions: The need for unplanned cardiac reintervention in neonates with critical congenital heart disease is strongly associated with increased mortality. Early unplanned reinterventions might be an important covariate in outcomes studies and useful as a quality improvement measure. (J Thorac Cardiovasc Surg 2013;145:671-7)

Hospital mortality remains an important issue for neonates undergoing complex cardiac surgical procedures. The mortality rates between institutions vary, indicating potential modifiable factors. Recent multicenter pooled data from the European Association for Cardiothoracic Surgery and the Society of Thoracic Surgeons Congenital Heart Surgery databases have indicated that the hospital mortality rates are approximately $8 \%$ after an arterial switch operation with ventricular septal defect repair, $14 \%$ after truncus arteriosus

\footnotetext{
From the Departments of Cardiology, ${ }^{\mathrm{a}}$ Cardiac Surgery, ${ }^{\mathrm{b}}$ and Pediatrics, ${ }^{\mathrm{c}}$ Children's Hospital Boston, Harvard Medical School, Boston, Mass; and Department of Biostatistics, ${ }^{\mathrm{d}}$ Harvard School of Public Health, Boston, Mass.

This study was funded in part by the Rochelle E. Rose Clinical Research Fund (to P.C.L.) and supported in part by a National Institutes of Health T32 training grant (to M.L.M.).

Disclosures: Authors have nothing to disclose with regard to commercial support.

Received for publication Sept 14, 2011; revisions received Feb 20, 2012; accepted for publication March 12, 2012; available ahead of print May 11, 2012.

Address for reprints: John M. Costello, MD, MPH, Department of Pediatrics, Northwestern University Feinberg School of Medicine, and Regenstein Cardiac Care Unit, Division of Cardiology, Ann \& Robert H. Lurie Children's Hospital of Chicago, 225 E Chicago Ave, Chicago, IL 60611 (E-mail: jmcostello@luriechildrens. org).

$0022-5223 / \$ 36.00$

Copyright $(2013$ by The American Association for Thoracic Surgery

doi: $10.1016 /$ j.jtcvs.2012.03.078
}

repair, and $25 \%$ after a stage 1 Norwood operation. ${ }^{1}$ Neonates are at risk of complex postoperative courses for numerous reasons, with contribution from multiple preoperative, intraoperative, and postoperative factors. ${ }^{2-7}$

We sought to further the understanding of neonates with significant residual or undiagnosed anatomic cardiac lesions that lead to an unplanned cardiac reintervention during the index hospitalization. Intuitively, such patients will be at risk of adverse outcomes. Determining whether a patient has a significant residual anatomic lesion might be challenging, because standard definitions do not exist and the decision to reintervene is dependent on clinical judgment and the clinical status of the patient at the assessment. Therefore, the focus of the present study was the occurrence of unplanned cardiac reinterventions. One aim of the present study was to describe the incidence and type of unplanned reinterventions in neonates with critical congenital heart disease. We also sought to identify the risk factors for unplanned reintervention, focusing on preoperative variables in an attempt to proactively characterize the infant at risk of unplanned reinterventions. Finally, we sought to explore the relationship between unplanned cardiac reinterventions and hospital mortality. 


\section{Abbreviations and Acronyms \\ $\mathrm{CI}=$ confidence interval \\ OR $=$ odds ratio \\ RACHS-1 = Risk Adjustment in Congenital Heart Surgery, version 1}

\section{METHODS}

This was a retrospective, single-center, cohort study. Data were obtained in part from a pre-existing research database that included all neonatal $(<28$ days of age) admissions to the cardiac intensive care unit at our institution. ${ }^{6,8}$ Additional variables were obtained from medical record review. We included all neonates admitted to our institution from January 1, 2002 to December 31, 2008 with critical congenital heart disease who had undergone cardiac surgery within the first 28 days of life. ${ }^{7}$ We excluded patients with critical congenital heart disease who had initially undergone interventional cardiac catheterization that was intended to allow discharge from the hospital without additional cardiac intervention. We also excluded patients with critical congenital heart disease who died or had had life support withdrawn before any cardiac surgery, premature neonates undergoing isolated patent ductus arteriosus ligation, and those whose initial cardiac surgical procedure was performed at another institution.

We assigned patients to the unplanned cardiac reintervention group who underwent at least 1 additional cardiac surgical operation or interventional cardiac catheterization during the same hospitalization as the initial cardiac operation. The operative procedures that did not directly address residual cardiac lesions, such as delayed sternal closure, extracorporeal membrane oxygenation cannulation, or diaphragm plication, were not considered unplanned cardiac reinterventions. Diagnostic cardiac catheterizations during which no intervention was performed were also not considered unplanned cardiac reinterventions. The comparison group consisted of all remaining neonates who did not require an unplanned cardiac reintervention.

From a review of the available data, we attempted to assign each patient's first cardiac reintervention to 1 or more of the following categories, adopted from those reported by Ma and colleagues' ${ }^{9}$ a residual anatomic defect, defined as correctly planned surgery but technically inadequate because of a residual anatomic problem; adequate surgical execution but an incomplete preoperative diagnosis; or a failed therapeutic plan. A failed therapeutic plan was defined as an adequately performed surgical procedure but that with the benefit of retrospective analysis, an alternative therapeutic plan might have been preferable. An unplanned reintervention was categorized as unpreventable if none of these categories were applicable (eg, surgery for acquired pulmonary venous stenosis in a patient without previous pulmonary vein surgery or thrombectomy of a systemic to pulmonary shunt without the need for additional surgical revision of the shunt).

Multiple preoperative covariates that could influence the need for unplanned cardiac reintervention or confound the association between the need for unplanned cardiac reintervention and mortality were assessed. These included nonreassuring fetal status just before delivery, prenatal diagnosis, delivery location, birth weight, birth weight that was small for gestational age, gender, major noncardiac birth defects, and the presence of chromosomal abnormalities. We recorded the exposure to, and duration of, cardiopulmonary bypass and when possible assigned each patient a Risk Adjustment in Congenital Heart Surgery, version 1 (RACHS-1), category. ${ }^{2}$ RACHS- 1 is a validated risk adjustment method that groups cardiac surgical procedures with similar expected in-hospital mortality rates into 6 predefined risk categories, in which category 1 has the lowest risk of death (eg, atrial septal defect closure) and category 6 the greatest risk of death (eg, stage 1 Norwood operation). Patients who underwent operations that could not be assigned a RACHS-1 category were analyzed as a separate group. The experience of the attending cardiac surgeon was calculated by subtracting the date that the surgeon first practiced as an attending congenital heart surgeon at any institution from the date of the operation. ${ }^{10}$ The Children's Hospital Boston Committee on Clinical Investigation approved the present study, and the requirement for informed consent was waived.

\section{Statistical Analysis}

Continuous variables are reported as the mean \pm standard deviation or median and range, as appropriate, and binary and categorical variables are reported as counts and percentages. The characteristics of the patients requiring unplanned cardiac reintervention were compared with those of the controls who did not using the Wilcoxon rank sum test or Fisher's exact test. The covariates associated $(P<.1)$ on univariate analysis with unplanned cardiac reintervention were considered for inclusion in a multivariate logistic regression model developed using manual backward stepwise selection. For the outcomes analysis, the characteristics of the patients who died were compared with those who did not using the Wilcoxon rank sum test or Fisher's exact test. Independent predictors of mortality were identified in a multivariate logistic regression model using a similar approach to that noted above. Statistical significance was achieved in the multivariate models when the $95 \%$ confidence intervals (CIs) for adjusted odds ratios (ORs) did not include $1(P<.05)$. The SAS System for Windows, version 9.2 (SAS Institute, Cary, NC), was used for statistical analysis.

\section{RESULTS \\ Study Patients}

During the 7-year study period, 1328 neonates with critical congenital heart disease were admitted to our institution. Of these neonates, we excluded 260 premature neonates undergoing isolated patent ductus arteriosus ligation, 8 who died of end-organ injury before any cardiac intervention, 6 with fatal syndromes or major additional malformations for whom life support was withdrawn before cardiac intervention, and 5 whose initial cardiac surgical procedure was performed at another institution. Neonates with critical congenital heart disease who underwent a primary catheter-based intervention $(n=106)$ were also excluded from the present analysis.

\section{Unplanned Cardiac Reinterventions}

A total of 943 neonates who met the study criteria and underwent cardiac surgery were included in the present study. Of these, $104(11 \%)$ underwent an unplanned cardiac reintervention, including 66 patients who underwent reoperation $(7 \%), 16$ who underwent interventional cardiac catheterization $(2 \%)$, and $22(2 \%)$ in whom both interventional cardiac catheterization and surgical reintervention were performed. The median interval between the initial cardiac surgical procedure and the first reintervention was 7 days (range, $0-140$ days). Of the 104 patients who underwent unplanned cardiac reintervention, the most common procedures involved manipulation of systemic to pulmonary shunts and pulmonary artery bands and correction of residual intracardiac shunts and aortic arch obstruction (Table 1). At the first unplanned reintervention, 35 patients $(34 \%)$ required extracorporeal membrane oxygenation, 51 $(49 \%)$ were receiving mechanical ventilation, $9(9 \%)$ were spontaneously breathing but in the cardiac intensive 
TABLE 1. Details of first unplanned cardiac reintervention $(n=104)$

\begin{tabular}{|c|c|c|}
\hline & Patients $(\%)$ & Other procedures at same reintervention \\
\hline \multicolumn{3}{|l|}{ Surgical reintervention } \\
\hline Revision of systemic-pulmonary artery shunt & $39(38)$ & \\
\hline Revision of pulmonary artery band & $10(10)$ & Atrial septectomy $(\mathrm{n}=1)$ \\
\hline Intracardiac shunt repair & $9(9)$ & \\
\hline Aortic arch augmentation & $9(9)$ & Aortic arch stent placement $(\mathrm{n}=1)$; tricuspid valvuloplasty $(\mathrm{n}=1)$ \\
\hline Surgical valvuloplasty & $4(4)$ & \\
\hline Pulmonary venous obstruction surgery & $3(3)$ & \\
\hline Right ventricular outflow tract obstruction surgery & $3(3)$ & \\
\hline Right ventricular pulmonary artery conduit placement/revision & $3(3)$ & Tricuspid valvuloplasty $(\mathrm{n}=1)$; VSD closure $(\mathrm{n}=1)$ \\
\hline Bidirectional Glenn procedure & $2(2)$ & $\begin{array}{l}\text { Pulmonary arterioplasty }(\mathrm{n}=2) \text {; DKS revision }(\mathrm{n}=1) \text {; tricuspid } \\
\quad \text { valvuloplasty }(\mathrm{n}=1)\end{array}$ \\
\hline Heart transplantation & $1(1)$ & \\
\hline DKS revision & $1(1)$ & \\
\hline Left ventricular outflow tract obstruction surgery & $1(1)$ & \\
\hline \multicolumn{3}{|l|}{ Catheter-based reintervention } \\
\hline Pulmonary artery balloon dilation & $8(8)$ & \\
\hline Aortopulmonary collateral coil occlusion & $3(3)$ & \\
\hline Balloon dilation of atrial septum & $3(3)$ & \\
\hline Systemic vein balloon dilation & $2(2)$ & \\
\hline Pulmonary artery band balloon dilation & $2(2)$ & \\
\hline Pulmonary valve balloon dilation & $1(1)$ & \\
\hline
\end{tabular}

care unit, and $9(9 \%)$ were on the cardiac ward. The most common clinical concerns preceding the first unplanned reintervention as noted in the medical record by the managing physicians were excessive cyanosis $(\mathrm{n}=36,35 \%)$, persistent or severe low cardiac output syndrome $(\mathrm{n}=18,17 \%)$, and excessive pulmonary blood flow related to a surgical systemic to pulmonary shunt $(\mathrm{n}=17,16 \%)$ or residual intracardiac shunt $(\mathrm{n}=11,11 \%)$.

After a review of the relevant data, the factors thought by the study investigators to be most contributory to an unplanned cardiac reintervention were a residual anatomic defect in 65 patients $(63 \%)$, adequate surgical execution but incomplete preoperative diagnosis in 5 patients $(5 \%)$, both of these factors in 1 patient $(1 \%)$, and adequate surgical execution but a failed therapeutic plan in 10 patients $(10 \%)$. In 18 patients $(17 \%)$, the need for unplanned reintervention could not be attributable to any of the study factors and was thought to be unpreventable. Of the 39 reinterventions involving reoperation on a systemic to pulmonary artery shunt, 17 (44\%) were isolated shunt thrombectomies that were deemed unpreventable, and 22 that involved revision of shunt size, location, or anastomosis, were categorized as residual anatomic defects. In 5 patients $(5 \%)$, the most contributory factor to the unplanned cardiac reintervention was unclear.

\section{Risk Factors for Unplanned Cardiac Reintervention}

A prenatal diagnosis of congenital heart disease, delivery at an adjacent institution, lower gestational age and birth weight, the need for mechanical ventilation before surgery, the presence of respiratory distress syndrome, a noncardiac structural anomaly, fewer than 9 years of attending surgeon experience (the lowest quartile of attending surgeon experience), and a greater RACHS-1 category were all significantly associated with a greater likelihood of an unplanned reintervention (Table 2). On multivariate analysis, a prenatal diagnosis of congenital heart disease, lower birth weight, the need for mechanical ventilation before cardiac intervention, fewer than 9 years of attending surgeon experience, and greater RACHS-1 category were independent risk factors for an unplanned cardiac reintervention (Table 3). In a secondary analysis that excluded 17 patients whose initial unplanned reoperation was a shunt thrombectomy, the same independent risk factors for unplanned reintervention were identified (Table 3), with the addition of the presence of a noncardiac structural anomaly (data not shown).

\section{Hospital Mortality: Rates and Risk Factors}

Of the 104 neonates who underwent an unplanned cardiac reintervention, 33 (32\%) died during the hospitalization. In contrast, of the 839 neonates who did not undergo an unplanned reintervention, $31(4 \%)$ died. Compared with the patients who did not undergo an unplanned reintervention, the overall unadjusted OR for death in patients undergoing an unplanned cardiac reintervention was 12.1 (95\% CI, 7.0-20.9; $P<.001$ ). Of those 31 patients who did not undergo reintervention and subsequently died, 4 $(13 \%)$ had residual anatomic lesions, of whom 2 were moribund when the residual lesion was identified and 2 had experienced a coronary artery injury during a Norwood 
TABLE 2. Univariate analysis of potential risk factors for unplanned cardiac reintervention in neonates who underwent cardiac surgery

\begin{tabular}{|c|c|c|c|}
\hline \multirow[b]{2}{*}{ Variable } & \multicolumn{2}{|c|}{ Reintervention } & \multirow[b]{2}{*}{$P$ value } \\
\hline & Yes $(n=104)$ & No $(\mathbf{n}=839)$ & \\
\hline $\begin{array}{l}\text { Prenatal diagnosis of congenital } \\
\text { heart disease }\end{array}$ & $67(64)$ & $379(45)$ & $<.001$ \\
\hline Multiple gestation & $5(5)$ & $36(4)$ & .80 \\
\hline Fetal distress & $9(9)$ & $37(4)$ & .08 \\
\hline Delivery at adjacent institution & $61(59)$ & $315(38)$ & $<.001$ \\
\hline Cesarean delivery & $44(42)$ & $317(38)$ & .39 \\
\hline Gestational age at birth (wk) & & & .004 \\
\hline Median & 38 & 39 & \\
\hline $10 \%-90 \%$ & $35-40$ & $36-40$ & \\
\hline Birth weight $(\mathrm{kg})$ & $2.97 \pm 0.70$ & $3.16 \pm 0.64$ & .005 \\
\hline $\begin{array}{l}\text { Small for gestational age }(<2 \mathrm{SD} \\
\text { below mean) }\end{array}$ & $6(6)$ & $25(3)$ & .14 \\
\hline Male gender & $63(61)$ & $488(58)$ & .67 \\
\hline Noncardiac structural anomaly & $16(15)$ & $79(9)$ & .04 \\
\hline Chromosomal anomaly & $8(8)$ & $64(8)$ & 1.0 \\
\hline $\begin{array}{l}\text { Preoperative mechanical } \\
\text { ventilation }\end{array}$ & $68(65)$ & $438(52)$ & .01 \\
\hline Respiratory distress syndrome & $8(8)$ & $26(3)$ & .04 \\
\hline Age at first cardiac operation (d) & & & .47 \\
\hline Median & 6 & 6 & \\
\hline $10 \%-90 \%$ & $3-17$ & $3-16$ & \\
\hline RACHS- 1 category & & & $<.001$ \\
\hline $1-2$ & $3(3)$ & $124(15)$ & \\
\hline $3-4$ & $47(45)$ & 495 (59) & \\
\hline $5-6$ & $45(43)$ & $192(23)$ & \\
\hline Not assignable & $9(9)$ & $28(3)$ & \\
\hline CPB time (min) & & & .15 \\
\hline Median & 134 & 124 & \\
\hline $10 \%-90 \%$ & $0-261$ & $0-185$ & \\
\hline $\begin{array}{l}\text { Attending surgeon experience (y) } \\
\text { (quartiles) }\end{array}$ & & & .005 \\
\hline$\geq 20$ & $20(19)$ & $210(25)$ & \\
\hline 14-19.9 & $20(19)$ & $222(26)$ & \\
\hline $9-13.9$ & $23(22)$ & $211(25)$ & \\
\hline$<9$ & $41(39)$ & $197(24)$ & \\
\hline
\end{tabular}

Data presented as $\mathrm{n}(\%)$ or mean $\pm \mathrm{SD}$, unless otherwise specified. $C P B$, Cardiopulmonary bypass; RACHS-1, Risk Adjustment in Congenital Heart Surgery, version 1; $S D$, standard deviation.

operation. Two additional patients $(6 \%)$ had undergone adequate surgical execution but had an incomplete preoperative diagnosis that directly affected the outcome. The other 25 patients died of a variety of complications. In these patients, a review of the available data did not suggest an undiagnosed or residual cardiac lesion.

In the 33 patients who underwent an unplanned reintervention and subsequently died, the median interval between initial surgery and death was 32 days (range, 1-128 days), and the median duration between from first reintervention and death was 18 days (range, 0-21 days). No differences were found in the hospital mortality rates among patients undergoing surgical reintervention $(23 / 66,35 \%)$ or transcatheter reintervention $(4 / 16,25 \%)$, or both $(6 / 22,27 \%$;
TABLE 3. Independent risk factors for unplanned cardiac reintervention in neonates who underwent cardiac surgery

\begin{tabular}{llc}
\hline \multicolumn{1}{c}{ Variable } & OR $(\mathbf{9 5} \% \mathbf{C I})$ & $\boldsymbol{P}$ value \\
\hline Prenatal diagnosis of congenital heart disease & $1.9(1.2-3.0)$ & .01 \\
Birth weight (per kg) & $0.7(0.5-0.99)$ & .04 \\
Preintervention mechanical ventilation & $2.1(1.4-3.4)$ & .001 \\
RACHS-1 category & & \\
1-2 & Reference & \\
$3-4$ & $3.2(0.96-10.5)$ & .06 \\
5-6 & $7.1(2.1-23.9)$ & .002 \\
Not assignable & $10.1(2.5-40.9)$ & .001 \\
Attending surgeon experience $<9$ y & $2.2(1.4-3.4)$ & $<.001$ \\
\hline CI, Confidence interval; OR, odds ratio; RACHS-1, Risk Adjustment in Congenital \\
Heart Surgery, version 1.
\end{tabular}

$P=.66$ ) (Figure 1). Of the 29 patients who underwent unplanned reintervention within 2 days of the initial surgical procedure, 6 died $(21 \%)$. In contrast, 10 of 26 patients died after the initial reintervention between postoperative days 3 and 7 (38\% mortality; OR, 2.4; 95\% CI, 0.7-7.6), 12 of 23 patients died after the initial reintervention between postoperative days 8 and 18 (52\% mortality; OR, $4.2 ; 95 \%$ CI, $1.2-4.1$ ), and 5 of 26 patients died after initial reintervention on or after postoperative day 19 (19\% mortality; OR, 0.9; 95\% CI, 0.2-3.4).

The results of univariate analyses of the potential risk factors for mortality are listed in Table 4 . The factors significantly associated with hospital mortality included a prenatal diagnosis of congenital heart disease, lower gestational age and birth weight, the need for mechanical ventilation before surgery, respiratory distress syndrome, greater RACHS-1 category, longer cardiopulmonary bypass time, and unplanned cardiac reintervention. The years of attending surgeon experience was not associated with hospital mortality. On multivariate analysis, the independent

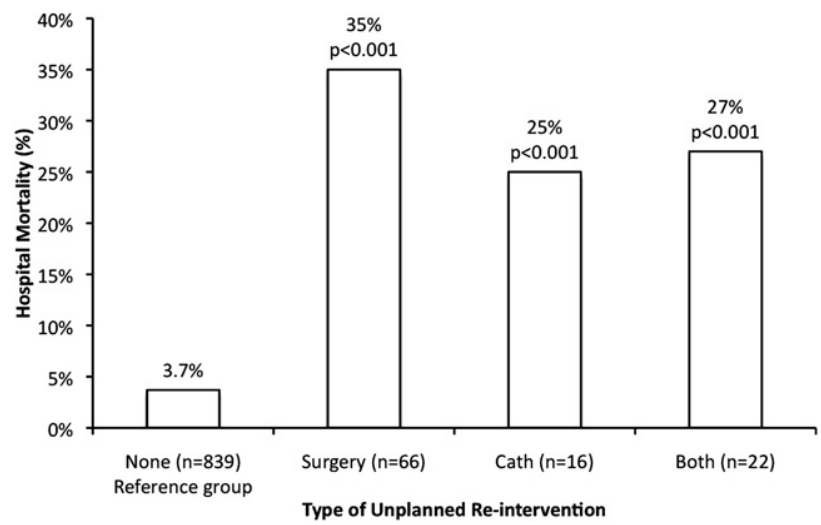

FIGURE 1. Hospital mortality rates in neonates undergoing cardiac surgery according to need for unplanned cardiac reintervention. $P$ values refer to comparisons of hospital mortality rates for those patients who required unplanned reintervention versus the reference group who did not require reintervention. 
TABLE 4. Univariate analysis of potential risk factors for hospital death in neonates who underwent cardiac surgery

\begin{tabular}{|c|c|c|c|}
\hline Variable & $\begin{array}{c}\text { Dead } \\
(\mathrm{n}=64)\end{array}$ & $\begin{array}{l}\text { Surviving } \\
(\mathbf{n}=\mathbf{8 7 9})\end{array}$ & $P$ value \\
\hline $\begin{array}{l}\text { Prenatal diagnosis of congenital } \\
\text { heart disease }\end{array}$ & $47(73)$ & $399(45)$ & $<.001$ \\
\hline Multiple gestation & $5(8)$ & $36(4)$ & .19 \\
\hline Fetal distress & $9(14)$ & 37 (4) & .003 \\
\hline Delivery at adjacent institution & $42(66)$ & $334(38)$ & $<.001$ \\
\hline Cesarean delivery & $35(55)$ & $326(37)$ & .007 \\
\hline Gestational age at birth (wk) & & & $<.001$ \\
\hline$>41$ & $3(5)$ & $40(5)$ & \\
\hline $39-40$ & $11(17)$ & $418(48)$ & \\
\hline $37-38$ & $22(34)$ & 297 (34) & \\
\hline $34-37$ & $17(27)$ & $99(11)$ & \\
\hline$<34$ & $11(17)$ & $25(3)$ & \\
\hline Birth weight $(\mathrm{kg})$ & $2.73 \pm 0.76$ & $3.17 \pm 0.63$ & $<.001$ \\
\hline $\begin{array}{l}\text { Small for gestational age } \\
\quad(<2 \mathrm{SD} \text { below mean })\end{array}$ & $1(2)$ & $30(3)$ & .72 \\
\hline Male gender & $36(56)$ & $515(59)$ & .79 \\
\hline Noncardiac structural anomaly & $10(16)$ & $86(10)$ & .14 \\
\hline Chromosomal anomaly & $4(6)$ & $68(8)$ & .81 \\
\hline Preoperative mechanical ventilation & $46(72)$ & $458(52)$ & .003 \\
\hline Respiratory distress syndrome & $10(16)$ & $24(3)$ & $<.001$ \\
\hline Age at first cardiac operation (d) & & & .93 \\
\hline Median & 6.5 & 6 & \\
\hline $10 \%-90 \%$ & $3-16$ & $3-16$ & \\
\hline RACHS- 1 category & & & $<.001$ \\
\hline $1-2$ & $2(3)$ & $125(14)$ & \\
\hline $3-4$ & $25(39)$ & 517 (59) & \\
\hline $5-6$ & $32(50)$ & $205(23)$ & \\
\hline Not assignable & $5(8)$ & $32(4)$ & \\
\hline CPB time (min) & & & $<.001$ \\
\hline Median & 145 & 124 & \\
\hline $10 \%-90 \%$ & $0-306$ & $0-182$ & \\
\hline CPB group (n) & & & .003 \\
\hline $0 \mathrm{~min}$ & $9(14)$ & $152(17)$ & \\
\hline $1-120 \mathrm{~min}$ & $9(14)$ & $248(28)$ & \\
\hline $121-150 \mathrm{~min}$ & $16(25)$ & $248(28)$ & \\
\hline$>150 \mathrm{~min}$ & $30(47)$ & $231(26)$ & \\
\hline Unplanned cardiac reintervention & $33(52)$ & $71(8)$ & $<.001$ \\
\hline $\begin{array}{l}\text { Attending surgeon experience } \\
\text { (quartiles) (y) }\end{array}$ & & & .17 \\
\hline$\geq 20$ & $12(19)$ & $218(25)$ & \\
\hline $14-19.9$ & $17(27)$ & 224 (26) & \\
\hline $9-13.9$ & $12(19)$ & $222(25)$ & \\
\hline$<9$ & $23(36)$ & $215(25)$ & \\
\hline
\end{tabular}

Data presented as $\mathrm{n}(\%)$ or mean $\pm \mathrm{SD}$, unless otherwise noted. $C P B$, Cardiopulmonary bypass; RACHS-1, Risk Adjustment in Congenital Heart Surgery, version 1; $S D$, standard deviation.

predictors of hospital mortality were a prenatal diagnosis of congenital heart disease, fetal distress just before delivery, lower gestational age at delivery, a RACHS-1 category of 5 to 6 , longer cardiopulmonary bypass time, and an unplanned cardiac reintervention (Table 5). Including all univariate predictors of mortality in the model had little effect on the association between unplanned cardiac
TABLE 5. Independent risk factors for hospital death in neonates who underwent cardiac surgery

\begin{tabular}{lcc}
\hline \multicolumn{1}{c}{ Variable } & Adjusted OR $(\mathbf{9 5} \%$ CI) & $\boldsymbol{P}$ value \\
\hline $\begin{array}{l}\text { Fetal distress } \\
\text { Gestational age at delivery (wk) }\end{array}$ & $2.8(1.1-7.2)$ & .03 \\
$\quad>41$ & $2.8(0.6-11.8)$ & .16 \\
$39-40$ & Reference & \\
$37-38$ & $2.5(1.3-5.5)$ & .02 \\
$34-37$ & $5.1(2.1-11.2)$ & $<.001$ \\
$<34$ & $16.9(6.0-48.1)$ & $<.001$ \\
RACHS-1 category & & \\
$1-2$ & Reference & \\
$3-4$ & $2.3(0.5-10.9)$ & .30 \\
5-6 & $6.3(1.3-30.6)$ & .02 \\
Not assignable & $3.8(0.6-24.6)$ & .16 \\
CPB time $>150$ min & $2.0(1.1-3.8)$ & .02 \\
Unplanned cardiac reintervention & $8.7(4.8-15.9)$ & $<.001$ \\
\hline CI, Confidence interval; $C P B$, cardiopulmonary bypass; OR, odds ratio; $R A C H S-1$, \\
Risk Adjustment in Congenital Heart Surgery, version 1. &
\end{tabular}

intervention and mortality (adjusted OR, 7.8; 95\% CI, $4.2-14.4 ; P<.001)$. In a secondary analysis that excluded 17 patients whose initial unplanned reoperation was shunt thrombectomy, the independent risk factors for hospital mortality were a prenatal diagnosis of congenital heart disease, a lower gestational age at delivery, a longer cardiopulmonary bypass time, and an unplanned cardiac reintervention (data not shown).

\section{DISCUSSION}

Neonates undergoing cardiac surgery are at greater risk of operative mortality compared with older children. The reasons are multifactorial but include the limited physiologic reserve of cardiac and other organ systems, smaller cardiac structures, greater procedural complexity, the potential for exaggerated interactions between the blood components and the cardiopulmonary bypass circuit, and the susceptibility to infection. ${ }^{11,12}$ Previous investigations have identified risk factors for mortality among neonatal cardiac surgical patients. The preoperative risk factors include lower gestational age at birth and lower weight at surgery and the presence of major noncardiac anomalies and genetic syndromes. ${ }^{3-6}$ The intraoperative risk factors include greater procedural complexity and a longer duration of cardiopulmonary bypass. ${ }^{1,2}$ Adding to that list, our study identified an unplanned reintervention after neonatal cardiac surgery as an additional risk factor contributing to mortality.

We found that an unplanned cardiac reintervention was performed in $11 \%$ of the study patients. That more than 1 in 10 neonates in a large and experienced cardiovascular program underwent 1 or more unplanned cardiac reinterventions calls attention to this important and understudied complication. The true incidence of important residual or 
undiagnosed lesions might be slightly greater; however, the use of an unplanned reintervention was chosen as the primary exposure variable to identify those lesions thought to warrant early cardiac reintervention as judged by the treating clinicians. The types of reintervention were quite heterogeneous.

\section{Unplanned Reinterventions and Mortality}

The overall hospital mortality rate for neonates undergoing cardiac surgery included in the present study was $7 \%$, with a disproportionate number of deaths occurring in patients who underwent an unplanned cardiac reintervention (32\% mortality). The strong association between unplanned reintervention and mortality persisted after adjustment for multiple confounders. We were unable to demonstrate a difference in mortality rates between surgical and transcatheter and both types of reintervention, suggesting that the circumstances prompting the reintervention might contribute more to mortality than the nature of the reintervention itself. With the exception of fetal distress, the other independent risk factors for mortality in the present study have all been previously described in neonates undergoing cardiac surgery. ${ }^{2-4}$

\section{Factors Contributing to Residual Lesions and Unplanned Reintervention}

We found that smaller neonates and those undergoing more complex procedures were at greatest risk of unplanned reintervention. There are likely nonmodifiable factors related to patient size that affect the technical aspects of cardiac surgery and limit the ability to easily assess for residual lesions. The association between a prenatal diagnosis of congenital heart disease and unplanned cardiac reintervention is difficult to explain, because the model controlled for surgical complexity using the RACHS- 1 categories. Unmeasured confounding variables, such as the heterogeneity of phenotypes within the individual RACHS-1 categories, might explain this association.

To describe the factors leading to unplanned reintervention, we attempted to assign each case to 1 of the following groups: a residual anatomic defect, an incomplete preoperative diagnosis, or a failed therapeutic plan. ${ }^{9}$ We found that residual anatomic defects appeared to be the most common factor leading to reintervention. Given the retrospective nature of the present study and the inherent marked variability within each type of congenital heart defect, unmeasured factors could have contributed to the occurrence of residual lesions despite optimal technical performance. Revision of systemic to pulmonary artery shunts was the most common type of unplanned cardiac reintervention. Of these 39 cases, $17(44 \%)$ were isolated shunt thrombectomies that were deemed to be unpreventable, and 22 involved revision of shunt size, location, or anastomoses and were categorized as residual anatomic defects.
Fewer years of attending surgeon experience was identified as a risk factor for unplanned cardiac reintervention but not mortality. The years of attending experience might not correlate closely with technical skill, and the overall case volume, specific case volume, and other factors might also be important. ${ }^{10} \mathrm{We}$ also recognize that the need for an unplanned reintervention is an imperfect measure of technical performance during the initial operation. Larrazabal and colleagues ${ }^{13}$ have proposed a more direct measure of the adequacy of repair after selected congenital heart surgical procedures. It was not feasible to use their technical performance scoring system in our investigation, because it was not applicable to many of the operations performed on our study patients. Although lower surgeon experience was a risk factor for unplanned reintervention, it was not associated with increased mortality. The reason for this discrepant finding is unknown. We speculate that more junior surgeons might have a lower threshold for early investigation of the adequacy of their repair, potentially leading to timelier intervention.

Only $5 \%$ of the unplanned cardiac reinterventions were thought to be attributable to an incomplete preoperative diagnosis. A previous study from our institution found that major diagnostic echocardiographic errors occurred in $1.9 \%$ of infants weighing more than $2.5 \mathrm{~kg}$ and $5.2 \%$ of infants weighing $2.5 \mathrm{~kg}$ or less. ${ }^{14}$ The risk factors for such diagnostic errors in our institution include rare diagnoses, greater anatomic complexity, and weight less than $5 \mathrm{~kg}{ }^{15}$ Awareness of these data might lead to improvements in diagnostic imaging and a reduced likelihood of reintervention for incomplete preoperative diagnoses. Ten percent of the unplanned reinterventions were undertaken because the initial therapeutic plan was failing in that the patient's clinical course was not following an expected trajectory despite a technically complete operation. At our institution, these outcomes are typically manifestations of an evolving clinical approach, such as attempts to perform early biventricular repair in neonates with borderline left heart structures. ${ }^{16}$

Decisions regarding the investigation of residual lesions and the need for, the ideal timing of, and the type of reintervention can be difficult clinical dilemmas. ${ }^{17,18}$ We found that patients who underwent unplanned reintervention within 2 days of the initial surgical procedure had lower hospital mortality than those who underwent reintervention 8 to 18 days after. A similar finding was noted in a study of infants and children who underwent unplanned cardiac catheterization early after cardiac surgery. ${ }^{17}$ It is likely patients undergoing reintervention within 2 days had overt signs of an anatomic problem or circulatory insufficiency that prompted early investigation. The increased mortality in patients undergoing unplanned reintervention between postoperative days 8 and 18 might represent the more difficult spectrum of patients whose clinical trajectory stalls. Such 
patients might experience cumulative morbidity from a prolonged stay in a critical care environment. The relatively low mortality rate in those patients undergoing reintervention after 19 or more days had elapsed since the initial operation might be the result of these patients having had less severe residual lesions. These data support the principle that when a postoperative patient is not following an anticipated clinical course, early evaluation should be considered. Cardiac catheterization should be considered for patients in whom noninvasive imaging fails to identify an etiology for hemodynamic or clinical compromise. ${ }^{19}$ The lower mortality in patients who underwent early reintervention suggests that rapid identification and correction of important residual anatomic lesions might lead to improved outcomes.

\section{Study Limitations}

The present study was limited by its retrospective, singlecenter design. The associations we identified between unplanned cardiac reinterventions and mortality do not prove causality. In some cases, unmeasured confounding variables might have contributed to the decision to proceed with reintervention rather than the presence of residual or unrecognized anatomic defects or a failed cardiac surgical therapeutic plan. There were no standardized management protocols for the evaluation of residual or unrecognized anatomic cardiac defects, and variations in the timing and modality of investigation likely existed among the clinicians. Our analysis of risk factors for mortality was also limited in that we only explored preoperative and selected intraoperative factors; thus, it is likely that additional intraoperative factors and postoperative care also contributed to the patient outcomes.

\section{CONCLUSIONS}

In our recent experience, $11 \%$ of neonates with critical congenital heart disease who underwent surgery underwent an unplanned cardiac reintervention during the same hospitalization. In addition to unplanned cardiac reintervention, fetal distress, greater RACHS-1 category, and longer duration of cardiopulmonary bypass were independent risk factors for hospital mortality. Our data support early evaluation to identify potential residual or unrecognized lesions in those neonates who are not following an expected clinical course after cardiac surgery, because early reintervention might lead to better outcomes. Patients at greatest risk of needing an unplanned reintervention can be identified preoperatively, providing an opportunity for programmatic modifications to reduce the occurrence of this complication. Additional work is necessary to determine whether there are modifiable technical and nontechnical factors that con- tribute to unplanned cardiac reinterventions, and whether this is a robust quality improvement metric.

\section{References}

1. O'Brien SM, Clarke DR, Jacobs JP, Jacobs ML, Lacour-Gayet FG, Pizarro C, et al. An empirically based tool for analyzing mortality associated with congenital heart surgery. J Thorac Cardiovasc Surg. 2009;138:1139-53.

2. Jenkins KJ, Gauvreau K, Newburger JW, Spray TL, Moller JH, Iezzoni LI. Consensus-based method for risk adjustment for surgery for congenital heart disease J Thorac Cardiovasc Surg. 2002;123:110-8.

3. Patel A, Hickey E, Mavroudis C, Jacobs JP, Jacobs ML, Backer CL, et al. Impact of noncardiac congenital and genetic abnormalities on outcomes in hypoplastic left heart syndrome. Ann Thorac Surg. 2010;89:1805-13.

4. Atz AM, Travison TG, Williams IA, Pearson GD, Laussen PC, Mahle WT, et al. Pediatric Heart Network Investigators. Prenatal diagnosis and risk factors for preoperative death in neonates with single right ventricle and systemic outflow obstruction: screening data from the Pediatric Heart Network Single Ventricle Reconstruction Trial. J Thorac Cardiovasc Surg. 2010;140:1245-50.

5. Curzon CL, Milford-Beland S, Li JS, O'Brien SM, Jacobs JP, Jacobs ML, et al. Cardiac surgery in infants with low birth weight is associated with increased mortality: analysis of the Society of Thoracic Surgeons Congenital Heart Database. J Thorac Cardiovasc Surg. 2008;135:546-51.

6. Costello JM, Polito A, Brown DW, McElrath TF, Graham DA, Thiagarajan RR, et al. Delivery before 39 weeks is associated with adverse outcomes in neonates with cardiac disease. Pediatrics. 2010;126:e277-84.

7. Schultz AH, Localio AR, Clark BJ, Ravishankar C, Videon N, Kimmel SE. Epidemiologic features of the presentation of critical congenital heart disease: implications for screening. Pediatrics. 2008;121:751-7.

8. Cheng HH, Almodovar MC, Laussen PC, Wypij D, Polito A, Brown DW, et al. Outcomes and risk factors for mortality in premature infants with critical congenital heart disease. Pediatr Cardiol. 2011;32:1139-46.

9. Ma M, Gauvreau K, Allan CK, Mayer JE, Jenkins KJ. Causes of death after congenital heart surgery. Ann Thorac Surg. 2007;83:1438-45.

10. Karamlou T, McCrindle BW, Blackstone EH, Cai S, Jonas RA, Bradley SM, et al Lesion-specific outcomes in neonates undergoing congenital heart surgery are related predominantly to patient and management factors rather than institution or surgeon experience: a Congenital Heart Surgeons Society study. J Thorac Cardiovasc Surg. 2010;139:569-77.

11. Wiegerinck RF, Cojoc A, Zeidenweber CM, Ding G, Shen M, Joyner RW, et al Force frequency relationship of the human ventricle increases during early postnatal development. Pediatr Res. 2009;65:414-9.

12. Costello JM, Graham DA, Morrow DF, Potter-Bynoe G, Sandora TJ, Laussen PC. Risk factors for central line-associated bloodstream infection in a pediatric cardiac intensive care unit. Pediatr Crit Care Med. 2009;10:453-9.

13. Larrazabal LA, Del Nido PJ, Jenkins KJ, Gauvreau K, Lacro R, Colan SD, et al. Measurement of technical performance in congenital heart surgery: a pilot study. Ann Thorac Surg. 2007;83:179-84.

14. Dorfman AL, Levine JC, Colan SD, Geva T. Accuracy of echocardiography in low birth weight infants with congenital heart disease. Pediatrics. 2005;115:102-7.

15. Benavidez OJ, Gauvreau K, Jenkins KJ, Geva T. Diagnostic errors in pediatric echocardiography: development of taxonomy and identification of risk factors Circulation. 2008;117:2995-3001.

16. McElhinney DB, Marshall AC, Wilkins-Haug LE, Brown DW, Benson CB, Silva V, et al. Predictors of technical success and postnatal biventricular outcome after in utero aortic valvuloplasty for aortic stenosis with evolving hypoplastic left heart syndrome. Circulation. 2009;120:1482-90.

17. Zahn EM, Dobrolet NC, Nykanen DG, Ojito J, Hannan RL, Burke RP. Interventional catheterization performed in the early postoperative period after congenital heart surgery in children. J Am Coll Cardiol. 2004;43:1264-9.

18. Asoh K, Hickey E, Dorostkar PC, Chaturvedi R, van Arsdell G, Humpl T, et al Outcomes of emergent cardiac catheterization following pediatric cardiac surgery. Catheter Cardiovasc Interv. 2009;73:933-40.

19. Feltes TF, Bacha E, Beekman RH III, Cheatham JP, Feinstein JA, Gomes AS et al. Indications for cardiac catheterization and intervention in pediatric cardiac disease: a scientific statement from the American Heart Association. Circulation. 2011;123:2607-52. 